\title{
Start of SuperKEKB
}

\author{
Y. Ohnishi*t \\ High Energy Accelerator Research Organization (KEK) \\ E-mail: vukiyoshi.onishi@kek.ip
}

The SuperKEKB B-Factory at KEK (Japan), after six years of the KEKB shutdown for the construction and renovation, has finally come to the Phase- 1 commissioning of the LER and HER rings, without the final focus system and the Belle II detector roll-in. Vacuum scrubbing, optics tuning, and beam related background measurements were performed in this phase. Low emittance tuning techniques have also been applied in order to set up the rings for Phase-2 with colliding beams next year. The status of the final focus system construction, as well as the schedule of the SuperKEKB project is also presented.

38th International Conference on High Energy Physics 3-10 August 2016

Chicago, USA

* Speaker.

$\dagger^{\dagger}$ on behalf of the SuperKEKB accelerator group 


\section{Luminosity}

The luminosity is the interaction rate per unit cross section for the colliding beams. The number of physics events is written by

$$
N=\int_{0}^{T} L \sigma d t
$$

where $\mathrm{L}$ is the luminosity, $\sigma$ is the cross section which is determined by a natural law, and $T$ is the duration of the experiment. The experiment of the particle physics is typically for $10-15$ years, which is shorter than the human lifetime. It is necessary to increase the luminosity when we try to acquire the huge number of physics events and to make a statistical error enough small. In the case of B meson production, the cross section, $\sigma$, is approximately $1 \mathrm{nb}$. When we try to observe a new physics via a rare decay or a forbidden reaction, $10-100$ times as high as the luminosity of KEKB is required since the cross section will be much smaller than $1 / 10-1 / 100$ of the $\mathrm{B}$ meson production. This approach to explore the new physics such as multiple Higgs bosons, origin of a flavor structure, and baryon-antibaryon asymmetry in the universe is the luminosity frontier.

Figure $\square$ shows the luminosity versus the center-mass energy for various colliders. There are two groups, one is the energy frontier collider and the other is the luminosity frontier collider. The luminosity of the energy frontier collider seems to be proportional to the beam energy. This implies that the collider experiments demonstrate that the analytic formula(Eq. [.2) which expresses that the luminosity is proportional to the Lorentz factor. However, the luminosity frontier collider achieves high luminosity compared with the energy frontier collider even though the beam energy is relatively lower than those colliders.

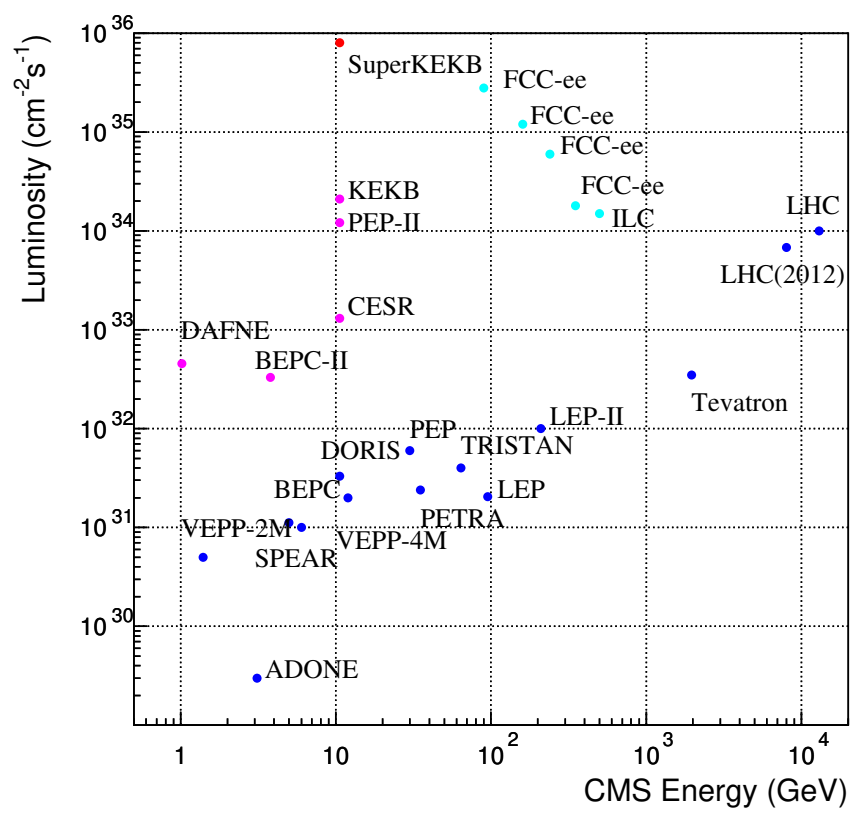

Figure 1: Luminosity versus energy of center-mass-system for various colliders. 
The SuperKEKB collider[四] is an asymmetric-energy and a double-ring electron-positron collider. The energy of the electron in the HER is $7 \mathrm{GeV}$ and the positron in the LER is $4 \mathrm{GeV}$, whose circumference is approximately $3 \mathrm{~km}$ for each ring. The collision point is one where the Belle II detector is roll-in. The target luminosity is $8 \times 10^{35} \mathrm{~cm}^{-2} \mathrm{~s}^{-1}$, which is 40 times as high as the predecessor KEKB collider[[2]. The luminosity formula can be expressed by

$$
L \propto \gamma \frac{I \xi_{y}}{\beta_{y}^{*}},
$$

where $I$ is the beam current, $\xi_{y}$ is the beam-beam parameter in the vertical direction, $\beta_{y}^{*}$ is the vertical beta function at the IP. In order to achieve 40 times as high luminosity as the KEKB collider, the vertical beta function at the IP is squeezed down to $1 / 20$ and the beam currents require the double with assuming the same vertical beam-beam parameter of KEKB. A "nano-beam scheme"[]] is adopted to accomplish the extremely high luminosity. Machine parameters of SuperKEKB compared with those of KEKB are shown in Table $\mathbb{W}$. A larger crossing angle and a small beam spot size in the both horizontal and vertical direction are applied in the nano-beam scheme. The crossing angle is $83 \mathrm{mrad}$ and the beam size is approximately $10 \mu \mathrm{m}$ in the horizontal direction. The nano-beam scheme can exchange the coordinate $x$ with $z$ when we consider the overlap region of two colliding beams:

$$
\begin{aligned}
& \tilde{\sigma}_{x}^{*}=\sigma_{z} \phi_{x} \\
& \tilde{\sigma}_{z}=\frac{\sigma_{x}^{*}}{\phi_{x}} \leq \beta_{y}^{*} \sim 300 \mu \mathrm{m},
\end{aligned}
$$

where the $\tilde{\sigma}_{x}^{*}$ and $\tilde{\sigma}_{z}$ are the effective horizontal beam size at the IP and the effective bunch length, respectively. Therefore, the vertical beta at the IP can be small down to be approximately $300 \mu \mathrm{m}$ without geometrical reduction of the luminosity due to an hour-glass effect although the bunch length $(\sim 6 \mathrm{~mm})$ is much longer than the vertical beta at the IP. This is one of the keys in the nano-beam scheme.

\begin{tabular}{lcccccc}
\hline & & \multicolumn{2}{c}{ KEKB } & \multicolumn{2}{c}{ SuperKEKB } & \\
& & LER & HER & LER & HER & Unit \\
\hline Beam-Beam Parameter & $\xi_{y}$ & 0.129 & 0.09 & 0.0881 & 0.0807 & \\
Beta Function at IP & $\beta_{y}^{*}$ & 5.9 & 5.9 & 0.27 & 0.30 & $\mathrm{~mm}$ \\
Beam Current & $I$ & 1.64 & 1.19 & 3.6 & 2.6 & $\mathrm{~A}$ \\
Horizontal Emittance & $\varepsilon_{x}$ & 18 & 24 & 3.2 & 4.6 & $\mathrm{~nm}$ \\
Vertical Emittance & $\varepsilon_{y}$ & 150 & 150 & 8.64 & 12.9 & $\mathrm{pm}$ \\
Vertical Beam Size & $\sigma_{y}^{*}$ & 940 & 940 & 48 & 62 & $\mathrm{~nm}$ \\
Crossing Angle & $2 \phi_{x}$ & \multicolumn{2}{c}{22} & \multicolumn{2}{c}{83} & $\mathrm{mrad}^{-2}$ \\
Luminosity & $L$ & $2.1 \times 10^{34}$ & $8 \times 10^{35}$ & $\mathrm{~cm}^{-2} \mathrm{~s}^{-1}$ \\
\hline
\end{tabular}

Table 1: Comparison of machine parameters for the final design of SuperKEKB with those of KEKB.

The famous luminosity formula which is the alternative expression of Eq. $\square 2$ is modified by replacing with the effective values as:

$$
L=\frac{N_{+} N_{-} f}{4 \pi \sigma_{x}^{*} \sigma_{y}^{*}}=\frac{N_{+} N_{-} f}{4 \pi \sigma_{z} \phi_{x} \sqrt{\varepsilon_{y} \beta_{y}^{*}}},
$$


where $N_{+}$and $N_{-}$are the number of particles of positrons and electrons, $f$ is the crossing frequency, $\varepsilon_{y}$ is the vertical emittance. On the other hand, the beam-beam parameters in the vertical and horizontal direction can be written by

$$
\begin{aligned}
& \xi_{y} \propto \frac{1}{\sigma_{z} \phi_{x}} \sqrt{\frac{\beta_{y}^{*}}{\varepsilon_{y}}} \\
& \xi_{x} \propto \frac{\beta_{x}^{*}}{\left(\sigma_{z} \phi_{x}\right)^{2}} \sim 0.003 .
\end{aligned}
$$

There is an upper limit for the beam-beam parameters which is typically smaller than 0.1 . When we make both the vertical emittance and the vertical beta at the IP small with keeping their ratio constant, the luminosity can be boosted. The horizontal beam-beam parameter is almost constant and very small in the nano-beam scheme even though the small emittance and the small beta at the IP are realized in the horizontal direction. Consequently, the dynamic beta and the dynamic emittance is small, then the aperture problem caused by the beam-size blowup does not occur.

\section{Schedule}

There are three stages for the commissioning of SuperKEKB. The initial commissioning without the final focus system and Belle II roll-in, Phase-1 has been finished. The Phase- 1 commissioning was started in February 2016 and operated until the end of June 2016 for about five months. The commissioning for Phase-2 will start in the late of 2017 or in the early of 2018 and will operate for 5 months with the final focus system and Belle II detector. The first collision will be performed during Phase-2, however the vertex detector will not be installed. Physics run with the full detector in Phase-3 will start in Autumn 2018, then the luminosity will increase gradually by squeezing the beta at the IP and increasing the beam currents toward the target luminosity. Figure $\square$ shows the

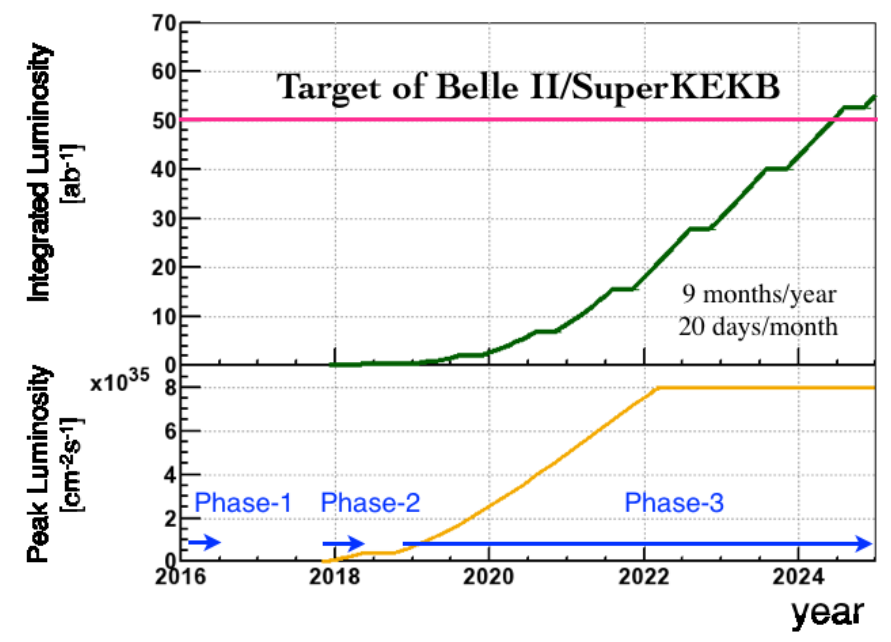

Figure 2: Estimation of the projected luminosity.

overall schedule and an estimation of the projected luminosity based on the latest status. The integrated luminosity will reach $50 \mathrm{ab}^{-1}$ until 2024 hopefully, whether this amount of data is enough or not for the sensitivity to the new physics is an open question. 


\section{Commissioning of Phase-1}

The vertical emittance is one of the most important issues in Phase- 1 as described above because the coupling parameter affects the luminosity performance in the nano-beam scheme significantly. The beam-size blowup induced by electron clouds in the LER is also an important issue in Phase-1. The study of the electron cloud was performed and confirmed mitigation techniques such as ante-chamber with TiN coating, and so on. The enough process of the vacuum scrubbing is necessary to reduce beam-related backgrounds for the detector. The Belle II group requires the beam dose of $360-720$ Ah during Phase-1. Figure B shows the history of 5 months during the Phase- 1 commissioning.

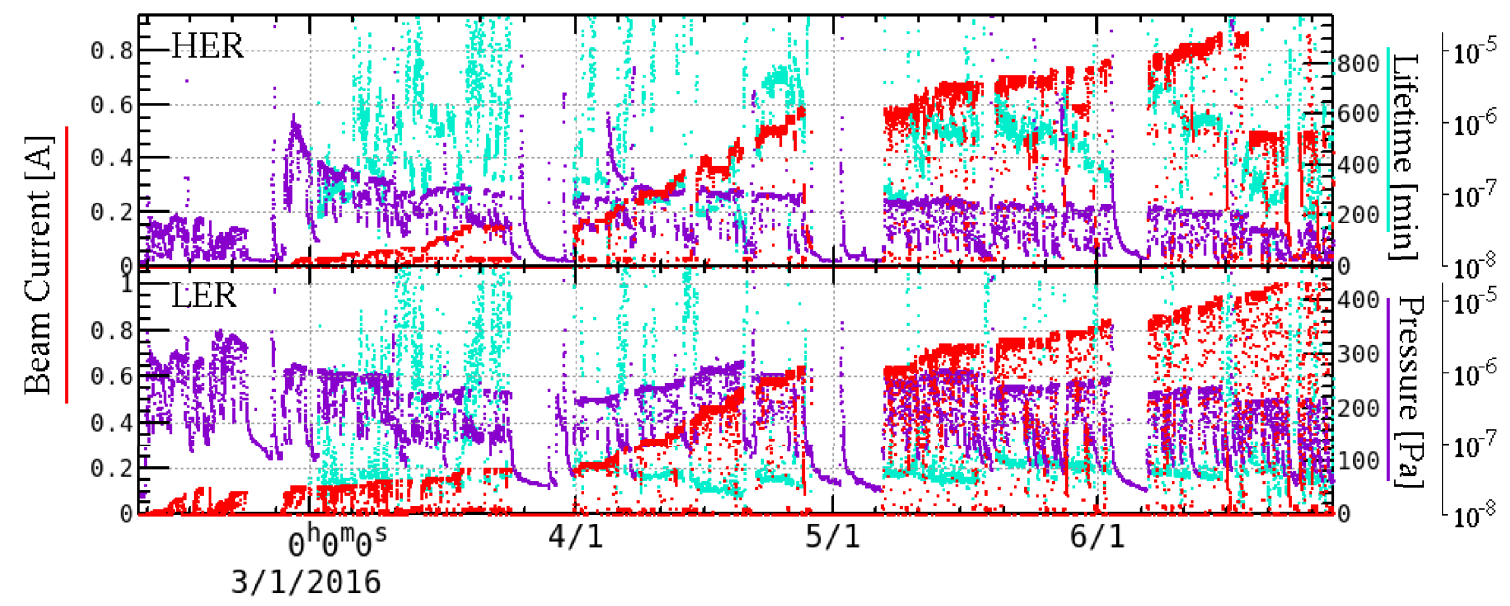

Figure 3: History of the Phase-1 commissioning.Beam current(red), lifetime(cyan), and average pressure(purple).

The RF system in the LER consists of normal conducting cavities with a HOM damped structure, ARES to store large beam current stably. A hybrid system of the ARES cavities and superconducting cavities(SCC) is utilized in the HER since the electron beam is not only large current but also high energy. The number of klystrons increases and the modification of the high-power input coupler is adopted in order to provide large beam power. The most of the components are reused from those of KEKB, however a relocation of the ARES cavities was performed to make an optimization of the RF system. The current upgrade of the RF is able to store up to $70 \%$ of the nominal beam current as shown in Table $\mathrm{W}$.

The vacuum system[ [i] in the LER has been newly designed to absorb intense synchrotron radiation emitted from positron beams and also suppress the electron cloud effect. Most of the vacuum pipes, about $93 \%$ out of the whole ring were replaced with new pipes. Ante-chambers made of an aluminum alloy with TiN coating were adopted in the arc section. On the other hand, most of the vacuum components were reused in the HER. The vacuum pipe made of a copper has a cross section of the race track in the arc section of the HER. Therefore, the vacuum scrubbing in the LER is necessary more than that of the HER. The beam dose of 780 Ah was achieved in the LER and $660 \mathrm{Ah}$ in the HER during Phase-1. The pressure rise has reached to be $8 \times 10^{-7} \mathrm{PaA}^{-1}$ in the LER and $4 \times 10^{-8} \mathrm{PaA}^{-1}$ in the HER, respectively. The pressure rise in the LER becomes 
approximately the same value as that of KEKB when we compare them at the same beam dose. It is found that the aluminum-alloy pipe with TiN coating achieves the same performance as the copper pipe if we consider the surface only. The pressure rise in the HER at the beginning of Phase-1 was smaller than that of the initial KEKB commissioning even though the vacuum pipes were exposed to the atmosphere for the upgrade work of the HER. It implies that the reused surface of the vacuum pipe keeps the condition of the past vacuum scrubbing during the KEKB operation.

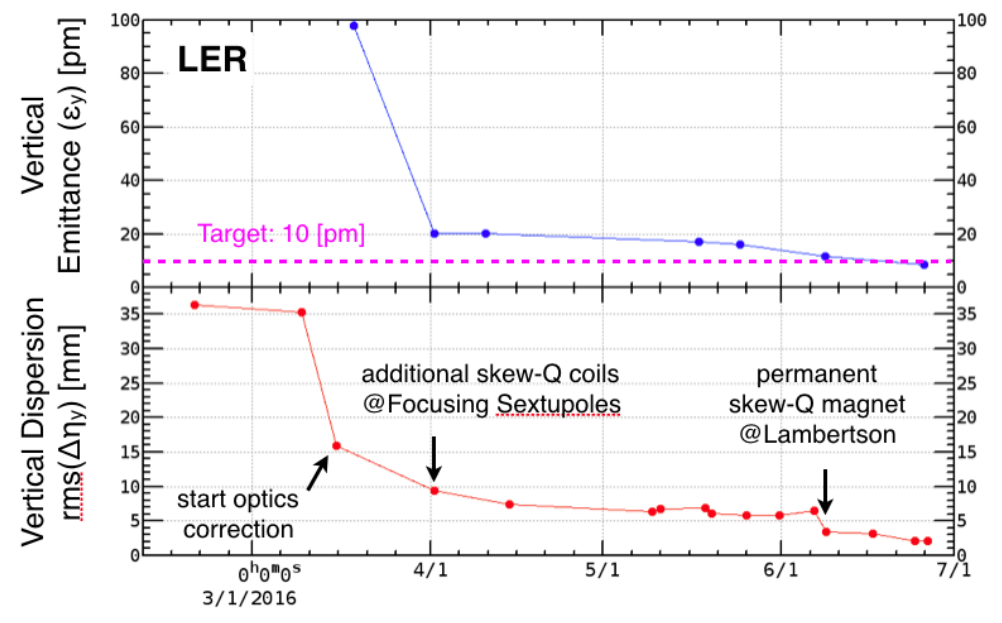

Figure 4: History of the vertical emittance and the vertical physical dispersion in the LER.

The lattice for Phase- 1 is the same lattice as those of Phase- 2 and Phase- 3 except for the interaction region(IR). The field gradient of the quadrupole magnets in the IR are adjusted so as to connect the arc lattice because the final focus magnets and the Belle II solenoid are not installed in Phase-1. The optics tuning without the final focus system, the solenoid field, and the local chromaticity corrections has been performed in Phase-1. The optical functions such as beta functions, dispersions, and X-Y couplings were measured and corrected in the LER and HER, respectively. The beta functions are obtained by orbit responses induced by six kinds of different dipole correctors for each $x$ and $y$ direction[[]]. The physical dispersions are measured by orbit displacements for the rf-frequency shift between $-500 \mathrm{~Hz}$ and $+500 \mathrm{~Hz}$. Note that the measured dispersions are physical dispersions and different from normal mode dispersions. In order to measure X-Y couplings, vertical leakage orbits from horizontal orbits are measured by using six kinds of different dipole correctors which correspond to the X-Y coupling parameters. The orbit distortions are measured by BPMs, the number of BPMs is 438 in the LER and 460 in the HER, respectively. The BPM gain mapping and the beam-based alignment have been performed before the optics tuning.

The measured beta functions and phase advances at BPMs are compared with those calculated from the model lattice and corrections of the field strength for each quadrupole families are obtained by fitting the measurements to the model. Correction coils installed for each quadrupole magnet are utilized to correct the beta functions. The X-Y couplings and the vertical dispersions are corrected by using skew quadrupole-like coils installed at each sextupole magnet. Since the arc lattice adopts non-interleaved sextupole correction scheme and two identical sextupoles are connected by $-I^{\prime}$ transfer matrix, the $\mathrm{X}-\mathrm{Y}$ couplings and the vertical dispersions can be corrected independently 
for each other. The horizontal dispersions are corrected by using asymmetric local bumps in the horizontal plane at a pair of two focusing sextupole magnets.

\begin{tabular}{lcccc}
\hline Items & Symbol & LER & HER & \\
\hline Coupling strength & $\left|C^{-}\right|$ & 1.2 & 2.0 & $\times 10^{-3}$ \\
X-Y coupling* & $\operatorname{rms}(\Delta y) / \mathrm{rms}(\Delta x)$ & 0.9 & 0.6 & $\%$ \\
Horizontal dispersion & $\operatorname{rms}\left(\Delta \eta_{x}\right)$ & 8 & 11 & $\mathrm{~mm}$ \\
Vertical dispersion & $\operatorname{rms}\left(\Delta \eta_{y}\right)$ & 2 & 2 & $\mathrm{~mm}$ \\
Horizontal $\beta$ function & $\operatorname{rms}\left(\Delta \beta_{x} / \beta_{x}\right)$ & 3 & 3 & $\%$ \\
Vertical $\beta$ function & $\operatorname{rms}\left(\Delta \beta_{y} / \beta_{y}\right)$ & 3 & 3 & $\%$ \\
Horizontal tune & $\Delta v_{x}$ & 2 & 5 & $\times 10^{-4}$ \\
Vertical tune & $\Delta v_{y}$ & 5 & 1 & $\times 10^{-4}$ \\
\hline
\end{tabular}

Table 2: Results of the Optics Tuning in Phase-1. X-Y coupling* refers an average value of $\operatorname{rms}(\Delta y) / \mathrm{rms}(\Delta x)$ induced by six kinds of horizontal dipole correctors. Dispersions are physical variables in the table.

Table $\square$ shows the results of optics corrections. After these optics corrections are applied, the vertical emittance has achieved to be $8 \mathrm{pm}$ in the LER which is measured by an X-ray beam size monitor. The vertical emittance is obtained from the vertical beam size by using $\varepsilon_{y}=\sigma_{y}^{2} / \beta_{y}$. Figure $\$$ shows the history of the vertical emittance and the vertical physical dispersion in the LER. In another way, the vertical emittance can be estimated by measured optical functions and magnet configurations:

$$
\varepsilon_{y}=C_{q} \gamma^{2} \frac{I_{5, y}}{I_{2}}
$$

where $C_{q}=3.84 \times 10^{-13} \mathrm{~m}$ and

$$
\begin{aligned}
I_{5, y} & =\int_{\text {Bend }} \frac{\gamma_{y} \eta_{y}^{2}+2 \alpha_{y} \eta_{y} \eta_{p y}+\beta_{y} \eta_{p y}^{2}}{|\rho|^{3}} d s \\
I_{2} & =\int_{\text {Bend }} \frac{1}{\rho^{2}} d s .
\end{aligned}
$$

The $\rho$ is the bending radius, $\alpha_{y}, \beta_{y}$, and $\gamma_{y}$ are Twiss parameters, $\eta_{y}$ and $\eta_{p y}$ are the vertical normal dispersions. The normal dispersions can be derived by X-Y coupling parameters and transfer matrix between neighboring two BPMs in the model. The vertical emittance of $8 \mathrm{pm}$ in the LER is also obtained by this estimation which is consistent with the X-ray measurement. On the other hand, the measured vertical beam size in the HER was $30 \mu \mathrm{m}$ for $\beta_{y}=7.6 \mathrm{~m}$ without any corrections, which corresponds to the vertical emittance of $40 \mathrm{pm}$ when corrections for the X-ray measurement was applied. This value seems to be too large because the optics tuning is the same level between the LER and HER as shown in Table $\square$. A simulation assuming a misalignment of the sextupoles which reproduces the measured optical functions provides the vertical emittance of $5-20 \mathrm{pm}$. We consider that the calibration issues of X-ray monitor still remains. The vertical emittance is indirectly estimated to be $6 \mathrm{pm}$ by using the measured optical functions in the HER. 


\section{Upgrade for Phase-2}

Major upgrade issue between Phase- 1 and Phase- 2 is a construction of the final focus system and a positron damping ring to keep enough injection aperture. The final focus system consists of four quadrupoles, 16 corrector coils, 4 cancel coils for the HER to compensate leakage field from the LER, and compensation solenoid for each side of the IP. These magnets are superconducting magnets and have iron or permendur yokes except for the most closest magnets to the IP in the LER. The left side of the final focus system has been installed in the IR and we have measured and adjusted an alignment of the magnets. The fabrication of the final focus system for the right side of the IP will be finished and installed within this year.

A flux concentrator and 10 large aperture S-band accelerating structures were installed to increase positron intensity for the LER. The huge emittance of the initial positron beams is decreased by the positron damping ring at $1.1 \mathrm{GeV}$ at the linac injector. The construction of the positron damping ring will be finished and the commissioning will start in the late of 2017.

\section{Conclusions}

The vacuum scrubbing was successfully done and the low emittance tuning was performed during Phase-1. The vertical emittance of $8 \mathrm{pm}$ which is smaller than the tentative target value at this stage has been achieved in the LER. The vacuum system, the RF system, and the beam instrumentation worked without any serious trouble. The fabrication and field measurements of the final focus system and the construction of the positron damping ring will be completed next year. The first collision will be provided in the end of 2017 or in the early of 2018 . The proof of the nano-beam scheme will be performed during Phase-2 and this is a challenge and very exciting. The countdown to the next generation B-factory toward more than $10^{35} \mathrm{~cm}^{-2} \mathrm{~s}^{-1}$ is already in progress.

\section{References}

[1] Y. Ohnishi et al., Accelerator Design of SuperKEKB, Prog. Theor. Exp. Phys. 201303 A011 (2013).

[2] T. Abe et al., Achievements of KEKB, Prog. Theor. Exp. Phys. 2013 03A001 (2013).

[3] SuperB Conceptual Design Report, INFN/AE-07/2, SLAC-R-856, LAL 07-15, March 2007.

[4] Y. Suetsugu et al., First Commissioning of the SuperKEKB Vacuum System, in Proceedings of IPAC2016, Busan, Korea, May (2016), paper TUOCB01.

[5] A. Morita et al., Measurement and correction of on- and off-momentum beta functions at KEKB, Phys. Rev. ST-AB, 10072801 (2007). 